\title{
THE THIRTY-FIRS'T ANNUAL MEETING OF THE SOCIETY
}

The thirty-first Annual Meeting of the American Mathematical Society was held at George Washington University, December 29-January 1, inclusive, in conjunction with the meetings of the Mathematical Association of America and the American Association for the Advancement of Science. Monday afternoon and Tuesday morning and afternoon were devoted to the regular scientific papers delivered before the Society. On Wednesday and Thursday mornings there were joint sessions with papers given by request; and on Wednesday and Thursday afternoons the Mathematical Association held its regular sessions. The meeting was brought to a conclusion by the dinner held jointly on Thursday evening with the Mathematical Association and Section A at the headquarters of the mathematicians, the Franklin Square Hotel. President Coolidge of the Mathematical Association presided at this function and some one hundred twenty-five were in attendance.

At the end of the Tuesday morning session it was voted to express to George Washington University and the department of mathematics the thanks of the Society for its cordial hospitality during this meeting, the largest in the history of the Society, and one of the most pleasant and most successful.

The attendance included the following one hundred fifty-five members of the Society:

C. R. Adams, O. S. Adams, R. B. Adams, Alexander, Archibald, G. N. Armstrong, Atchison, Bacon, Barney, W. J. Berry, Birkhoff, Bliss, Borden, E. W. Brown, H. S. Brown, Bruce, J. A. Bullard, W. G. Bullard, Cairns, Cajori, Caldwell, Carmichael, Chapelon, Chittenden, Clements, Clutz, Abraham Cohen, Coolidge, Currier, J. E. Davis, Denton, Dickinson, Dillingham. Dresden, Eiesland, English, Erwin, Everett, Feinler, Finkel, Fite, Foberg, Focke, Fry, Garretson, Gehman, Gerst, Glenn, Grant, Gravatt, L. M. Graves, Guggenbühl, Hancock, J. G. Hardy, G. A. Harter, Hawkesworth, Heal, E. R. Hedrick, Archibald Henderson, Robert Henderson, Hickson, Hildebrandt, Hille, Himwich, Hodgkins, Hughes, Hulbert, Hurwitz, Ingels, Karpinski, Kennelly, Kindle, Kline, W. D. Lambert, 
Landry, Latimer, Lefschetz, Lehr, F. P. Lewis, Lunn, W. D. MacMillan, Manson, Mathewson, B. I. Miller, J. A. Miller, Mirick, H. H. Mitchell, C. N. Moore, R. L. Moore, Frank Morley, C. O. Morris, Marston Morse, F. R. Moulton, Murnaghan, F. H. Murray, Musselman, C. A. Nelson, Olds, Packer, Pell, F. W. Perkins, Plant, Rainich, Ramler, W. W. Rankin, Ranum, Rawlins, Remick, F. G. Reynolds, J. N. Rice, R. G. D. Richardson, H. L. Rietz, Ritt, Robert, G.E. Robinson, E. D. Roe, Roever, Root, Safford, J. B. Scarborough, Schoonmaker, Seely, Seidlin, Sharpe, Shenton, Siceloff, Silverman, 'T. McN. Simpson. Sinclair, Slaught, C.E. Smith, D. E. Smith, S. E. Smith, W. M. Smith, Synge, J. S. Taylor, J. M. Thomas, B. M. Turner, Tyler, Uhler, Van Orstrand, Veblen, J.L. Walsh, Wernicke, Whited, Widder, Wiener, Wiggin, A.H.Wilson, E.W.Wilson, Woodard, Woolard, Wyant, J. W. Young.

The following organizations were elected to Sustaining Membership :

Connecticut General Life Insurance Company;

Phoenix Mutual Life Insurance Company;

Sargent and Lundy, Construction Engineers.

The Council elected the following twenty-six persons to ordinary membership in the Society:

Mr. William Clarke Arnold, DePauw University;

Mr. Clyde Myron Cramlet, University of Washington;

Mr. Haskell Brooks Curry, Harvard University;

Mr. John H. Evans, Ohio National Life Insurance Company;

Miss Beatrice Alethea Fenner, Palo Alto;

Professor Robert Edwin Gaines, University of Richmond;

Mr. R. L. Grossnickle, University of Minnesota;

Mr. George Pryor Harmount, East High School, Columbus;

Professor Clyde Monroe Hobart, Northern Illinois State Teachers College;

Professor Theodore Jesse Hoover, Stanford University;

Mr. Luther William Hussey, Union College;

Mr. Arthur R. Jerbert, University of Washington;

Miss Myra I. Johnson, New York City;

Mr. Burton Wadsworth Jones, Western Reserve University;

Mr. John Joseph McCarthy, New York University;

Miss Muriel L. Metz, University of Cincinnati;

Professor Allan Benton Morton, Georgia School of Technology;

Professor William Pinkerton Ott, University of Alabama;

Mr.Paul E.Remington, Mountain States Telephone and Telegraph Company;

Dean Jonathan U. Rogers, Piedmont College;

Mr. Sergei Alexander Schelkunoff, Western Electric Company;

Professor Walter Francis Shenton, United States Naval Academy;

Mr. George W. Sublette, Minneapolis;

Mr. Walter Phillips Suesman, Providence;

Mr. William Herman Thurow, Technische Hochschule, Charlottenburg;

Miss Mabel Gertrude Whiting, Santa Ana Junior College.

The following twenty-six persons were elected to membership as nominees of sustaining members:

Mr. Horace Richardson Bassford, Metropolitan Life Insurance Company;

Mr. Lewis Warrington Chubb, Westinghouse Company;

Mr. Frank Conrad, Westinghouse Company;

Mr. Walter Dietz, Westinghouse Company; 
Mr. George Myron Eaton, Westinghouse Company;

Mr. Robert D. Evans, Westinghouse Company;

Mr. Laurence R. Golladay, Westinghouse Company;

Mr. Clinton Richards Hanna, Westinghouse Company;

Mr. William J. Hudson, Westinghouse Company;

Mr. Robertson G. Hunter, Equitable Life Insurance Company of Iowa;

Mr. Philip C. Irwin, Equitable Life Insurance Company of Iowa;

Mr. Charles Jablow, Westinghouse Company;

Mr. Edwin W. Kopf, Metropolitan Life Insurance Company;

Mr. Reginald Campbell McCankie, Equitable Life Insurance Company of Iowa;

Mr. W. D. MacKinnon, Equitable Life Insurance Company of Iowa;

Mr. Samuel Milligan, Metropolitan Life Insurance Company;

Mr. Henry Scholte Nollen, Equitable Life Insurance Company of Iowa;

Mr. J. Ormondroyd, Westinghouse Company;

Mr. Robert James Piersol. Westinghouse Company;

Mr. Jonathan Gumersol Sharp, Metropolitan Life Insurance Company;

Mr. C. E. Skinner, Westinghouse Company;

Mr. Francis M. Smith, Metropolitan Life Insurance Company;

Mr. Carl Richard Soderberg, Westinghouse Company;

Mr. Hans Christian Specht, Westinghouse Company;

Mr. Dayton Ulrey, Westinghouse Company;

Mr. D. I. Vinogradoff, Westinghouse Company.

The ordinary membership in the Society is now 1477, including 94 nominees of sustaining members and 81 life members. There are also 19 sustaining members the total amount of whose annual dues is $\$ 3650$. The total attendance of members at all meetings, including sectional meetings, during the past year was 509; the number of papers read was 231 . The number of members attending at least one meeting was 365. At the annual election 210 votes were cast.

The reports of the Treasurer and the auditors (Mr. S. A. Joffe and Professor Tomlinson Fort) were received, showing a balance of $\$ 11,370.66$, exclusive of special funds; of this, $\$ 5506.54$ is reserved to secure the life memberships, and \$2500.54 for publications for 1924 not yet completed. Sales of the Society's publications during the year amounted to $\$ 4938.58$.

The Committee on Endowment announced that, including the pledges extending over five years, the Endowment Fund stands at approximately $\$ 60,000$, of which $\$ 35,000$ is paid in and invested.

The Board of Trustees adopted a budget for 1925, 
showing estimated expenditures and receipts as $\$ 16,072$ and $\$ 15,400$ respectively; the deficit can be met from the cash surplus brought forward from earlier years.

The Librarian reported that the Library of the Society now contains 6733 volumes. This library has more than doubled in size since the last catalogue was published, in 1910; it is proposed to issue a new catalogue in 1925 .

At the annual election, which closed on Tuesday morning, the following trustees and officers and other members of the Council were chosen:

Board of. Trustees, Professor G. D. Birkhoff, Professor L. P. Eisenhart, Professor W. B. Fite, Mr. Robert Henderson, Professor R. G. D. Richardson.

President, Professor G. D. Birkhoff.

Vice-President, Professor G. C. Evans.

Assistant Secretary, Professor Arnold Dresden.

Member of the Editorial Committee of the Bulletin, Professor E. R. Hedrick.

Member of the Editorial Committee of the Transactions, Professor H. H. Mitchell.

Members of the Council, Dr. G. A. Campbell, Professors E. W. Chittenden, A. J. Kempner, H. E. Slaught, Virgil Snyder.

The tellers appointed by President Veblen to count the ballots were Professor J. F. Ritt, Dr. C. R. Adams, and Dr. Caroline Seely.

At the meeting of the Council, Professor L. P. Eisenhart was named to succeed himself as representative of the Society on the National Research Council. Professors Arnold Dresden and E. B. Stouffer were appointed to represent the Society on the Council of the American Association for the Advancement of Science.

It was voted to hold the Annual Meeting for 1925 in New York City, and that for 1926 in Philadelphia. 
Statistics were presented by the Committee on Membership which show that of the approximately twenty-seven hundred teachers of mathematics in the colleges of the United States and Canada only one-third are members of the Society. In the past four years the membership of the Society has been doubled. A resolution was passed thanking Professor Clara E. Smith for her untiring efforts in connection with the membership campaign.

An account of the proceedings connected with the presentation of the Bôcher Memorial Prize appears on page 193 of this issue.

The second Josiah Willard Gibbs Lecture was delivered on Tuesday evening by Mr. Robert Henderson, vice-president of the Equitable Life Assurance Society. This lecture, which appears in full in the present number of this BuLLETIN, was entitled Life insurance as a social service and as a mathematical problem. Professor James Pierpont has been invited to deliver the next lecture of this series.

At the joint session of the Society, the Mathematical Association of America, and Section A, held on Wednesday morning, the following papers were read:

I. Remarks on the foundations of geometry, by Professor Oswald Veblen, retiring President of the Society. This paper appeared in full in the March-April number of this BULLETIN.

II. The foundations of the theory of algebraic numbers, by Professor Harris Hancock, retiring Vice-President of Section A.

The program of the joint session held on Thursday morning with the Mathematical Association and Sections $\mathrm{A}, \mathrm{B}$, and $\mathrm{D}$ was as follows:

I. Stellar evolution, by Professor H. N. Russel. (Address delivered at the request of Section D.)

II. Is the universe finite? by Professor Archibald Henderson. (Address delivered at the request of the Mathematical Association of America.) 
Titles and abstracts of the papers read at the regular sessions of the Society follow below. Retiring President Veblen presided at the sessions of Monday afternoon and Tuesday morning, relieved by Professor Coolidge. President Birkhoff presided at the Tuesday afternoon session. The papers of Adams (second paper), Bennett, Church, Dodd, Franklin, Graustein, Hazlett, Hurwitz (third paper), Kasner, Lubben, MacNeish, R. L. Moore (second paper), Ritt, Stone, Vandiver, Wiener (second paper), Wilder, and Zeldin were read by title; Mr. Merriman's paper was read by Professor C. N. Moore. Professor de la Vallée-Poussin was introduced by the Secretary.

1. Mr. H. S. Vandiver: On methods for finding factors of large integers. Second paper.

In the author's first paper with this title (this Bulletis, vol. 30, p. 542) three new methods were described for factoring integers greater than eleven million. The present paper contains further details and examples in connection with the use of two of these methods. They are applied to the factorization of $13,179,643$ and $236,364,091$.

2. Mr. H. S. Vandiver: Note on trinomial congruences and the first case of Fermat's last theorem.

This note contains the proofs of some theorems concerning the relation

$$
x^{p}+y^{p}+z^{p}=0,
$$

$x, y$ and $z$ being integers prime to the odd prime $p$, that depend on the possibility of finding prime integers $q$ such that

$$
\xi^{p}+\eta^{p}+\zeta^{p} \equiv 0 \quad(\bmod q)
$$

has no solution in integers $\xi, \eta$ and $\zeta$ prime to $q$. In particular the following result is obtained: If (2) has no solutions under the conditions above mentioned, $q=1+m p$, and $m<10 p$, then (1) has no solutions in integers prime to $p$.

3. Mr. H. S. Vandiver: Summary of results and proofs concerning Fermat's last theorem.

This paper contains several results regarding Fermat's last theorem obtained by the author within the last ten years and not hitherto published. The proofs are indicated. 
4. Mr. G. Y. Rainich: Stereographic representation of surfaces.

Given a fixed plane $\boldsymbol{I I}$, a fixed point $P$ outside $\boldsymbol{I I}$ and a surface $\Sigma$, we represent a point of $\Sigma$ by the intersection with $\boldsymbol{I}$ of the bisector of the angle formed at $P$ by the perpendiculars to $\Pi$ and to the tangent plane of $\Sigma$ at the point represented. If we take cartesian coordinates of the representing point for parameters of the point represented, the surface can be determined by its second differential form; given the coefficients of this form, which must satisfy the Codazzi equations, we can find the parametric expression of the surface in the shape of three definite integrals. The Weierstrass representation of a minimal surface is a special case of this. The Codazzi equations being linear, we obtain a new solution by adding two known solutions; from the representations of a sphere and a minimal surface we obtain in this way a representation of surfaces of constant mean curvature. The method can be applied to hypersurfaces immersed in a flat space of next higher dimensionality. This is a particular case of the "modified tensor analysis" previously indicated by the author.

5. Mr. G. Y. Rainich: On integrals in curved space.

If a tensor field of rank $r+s$ in a flat space is alternating in $r$ of its indices, an $r$-ple integral can be formed over an $r$-spread and this integral gives a tensor of rank $s$; but if the space in which the tensor field is considered is curved, the integration cannot be performed remaining in this space. In this case we choose $s$ arbitrary vectors in the containing flat space, and form at each point of the curved space the inner product of the tensor field at this point and the projections on the corresponding tangent bundle of the $s$ vectors of the containing space. In integrating the resulting tensor field of rank $r$ over an $r$-spread in the curved space we obtain a multilinear function of $s$ arbitrary vectors, i. e., a tensor of rank $s$, in the containing space. Conditions are discussed under which this tensor vanishes for a closed spread. Application is made of the foregoing in the cases of the indicator, the Riemann tensor, and the contracted Riemann tensor.

6. Miss Marguerite Lehr: The plane quintic with five cusps.

A modified form of del Pezzo's equation for this quintic suggests study of the system of conics through four cusps. 
This method gives a parametric expression for the curve, involving only a quadratic irrationality, establishes the fact that the curve is unipartite, and that its five cusps and five inflexions occur alternately, and gives the equations of the inflexional tangents. The inflexions, inflexional tangents, cuspidal tangents and a number of points of interest on the curve are obtained from the five given cusps by linear constructions.

7. Professor Anna J.Pell: A hyperbolic differential equation.

It is shown by a special example that the spectrum associated with a hyperbolic differential equation with a boundary condition may be continuous.

8. Professor C. N. Moore: On the application of Borel's method to the summation of Fourier's series.

In the present paper it is shown that for the summation of Fourier's series Borel's method is less effective than Cesàro's. This is accomplished in two ways, first by a direct study of the kernel of the singular integral which arises from the application of Borel's method to such series, and second by exhibiting examples of continuous functions whose Fourier development cannot be summed by this method. On the other hand it is shown that while the Lebesgue constants corresponding to the application of Borel's method become infinite of the same order as the ordinary Lebesgue constants, it is not true, as one might expect, that the Borel method is no more effective than ordinary convergence in the study of Fourier's series. For it is possible to set up examples of continuous functions whose Fourier development is divergent at a certain point but which can nevertheless be summed there by Borel's method.

9. Mr. G. M. Merriman: On a theorem concerning the convergence of triple series known to be summable $(C 1)$ and its application to the triple Fourier series.

In a paper presented to the Society, December, 1923, the author (1) proved a theorem deriving sufficient conditions for the convergence of double series from known results regarding their summability, and (2) applied this theorem to the double Fourier series, obtaining, as sufficient conditions for its convergence, generalizations of the Dini condition for the convergence of the simple Fourier series. 
The present paper contains generalizations to three variables of both of these theorems: (1) a theorem giving sufficient conditions for the convergence of triple series summable (C1), and (2) an application of this theorem to obtain conditions for the convergence of a triple Fourier series. These latter are again generalizations of the Dini condition.

10. Dr. L. M. Graves: The derivative as independent function in the calculus of variations.

This paper contains some extensions of the results presented by the author under the same title at the meeting of the Society in Chicago, April, 1924. The integrals I considered are of the form

$$
I=\int_{x_{1}}^{x_{2}} f\left(x, y_{1}+\int_{x_{1}}^{x} z d x, z(x)\right) d x .
$$

Admissible functions $z(x)$ are those which are bounded and integrable in the sense of Lebesgue. A necessary condition corresponding to that of Euler that an admissible function furnish a minimum for the integral $I$ is deduced on the basis of very weak restrictions on the function $f$. Application of this theorem to the second variation yields a necessary condition corresponding to that of Jacobi for an admissible function to minimize $I$. Sufficient conditions are obtained that a function $z(x)$ continuous almost everywhere furnish a minimum for the integral $I$ relative to the class of all admissible functions. The proofs are made without reference to any values of $x$ outside the interval $\left(x_{1}, x_{2}\right)$.

11. Dr. L. M. Graves: On functionals defined implicitly.

Lamson* has obtained a general implicit function theorem, but gives no information about the differentiability of the solutions of the functional equations he considers, which are of the two forms $y=F[y, z]$, and $G[y]=z$. In Part I of the present paper Lamson's classes of functions $[y]$ and $[z]$ are replaced by abstract sets concerning which the necessary properties are postulated, and conditions are given which ensure the existence of a solution $y=Y[z]$ of equations of the general type $G[y, z]=0$ in the neighborhood of an initial solution $\left(y_{0}, z_{0}\right)$. Further theorems are obtained concerning the existence of differentials and difference functions of all orders for the solutions $Y[z]$. In Part II a generali-

* American Journal, vol. 42, p. 243. 
zation of the imbedding theorems for solutions of systems of ordinary differential equations is obtained from the theorems of Part I by considering sets whose elements are functions on a composite range. In Part III a number of special cases are discussed.

12. Dr. S. D. Zeldin: The adjoint group in function space.

In a number of papers published since 1911, mainly by G. Kowalewski, the Lie theory of continuous groups has been applied to function space. The author extends the notion of the adjoint group to problems in function space, and determines its relation to its isomorphic group.

13. Professor J. R. Musselman: A configuration in space.

Starting with a triangle in a plane, the author constructs a configuration of 18 points and 36 lines. Connected with it is a dual configuration of 36 points and 18 lines. The points fall naturally into 4 sets of three 3 -points each; the whole is invariant under a ternary $G_{216}$, not the Hesse $G_{216}$. With a tetrahedron in space we are led to a configuration of 54 lines, 108 points and 108 planes such that 15 points are on each plane and 15 planes on each point. The lines fall into 4 sets of 27 each, such that on each set is a cubic surface of the Eckhardt type where the lines meet by threes in 18 points.

14. Professor H. F. MacNeish: A nomogram in n-dimensional space for the solution of n linear simultaneous equations.

In this paper a method is developed for solving $n$ linear equations in $n$ unknowns which is a generalization of the nomogram in the plane for the solution of 2 linear equations in 2 unknowns. This generalization is accomplished by the method of antisymmetric ratios considered in a paper Theorems concerning transversals of the $(n+1)$-hedron in $n$-dimensional space previously presented to the Society by the author.

15. Professor W. C. Graustein: Semi-parallel maps of surfaces.

If a map of two surfaces is called "parallel" it is assumed, in general, that there exist two systems of curves, one on each surface, having the property that their curves correspond and have at corresponding points parallel tangents. 
A map is given the name "semi-parallel" if there exist merely two families of curves, one on each surface, which have this property. The general semi-parallel map and important special cases are discussed in this paper.

16. Professor W. C. Graustein: An invariant of a general transformation of surfaces.

If two surfaces are in one-to-one correspondence, there exists between the pencils of tangent lines at corresponding points a projective correspondence. The two pencils cut the line of intersection of their planes in two projective ranges of points. The invariant cross ratio of this projectivity is introduced as an invariant of the transformation of the one surface into the other. Applications of it are made to the so-called fundamental or $F$ transformations.

17. Professor O. E. Glenn: On the residuation of systems of binary forms.

The two-dimensional system $Q_{1}$ of integers generated from an infinite series $S(\alpha): \alpha_{1}, \alpha_{2}, \cdots$, as first column, in which any element is $e=x \gamma+y \delta$, where $\delta$ is the element immediately preceding $e$ in the row and $\gamma$ the one next beneath $\delta$ in the column, has, for its general element, the binary form $\left(\alpha_{v-u+1}, \alpha_{v-u+2}, \cdots, \alpha_{v}\right)(x, y)^{u-1}$. This paper is a study of the residue system $Q$ of $Q_{1}$, modulo $n$. Subjects treated are generation of periodic sequences $S(\alpha)$ by means of periodic functions, period parallelograms and drift lines, prime elements. Some of the periodicities considered are manifestations of Fermat's theorem, Euler's function. $\varphi(n)$, and the theory of exponents.

18. Mr. C. G. Latimer: The arithmetics of generalized quaternion algebras.

The author considers the algebra over the field of rational numbers with the units $e_{0}=1, e_{1}, e_{2}, e_{3}$ and the following multiplication table: $e_{i} e_{0}=e_{0} e_{i}=e_{i}(i=0,1$, 2, 3), $e_{1}^{2}=-\alpha, e_{2}^{2}=-\beta, e_{3}^{2}=\alpha \beta, e_{1} e_{2}=-e_{2} e_{1}=e_{3}$, $e_{1} e_{3}=-e_{3} e_{1}=\alpha e_{2}, e_{2} e_{3}=-e_{3} e_{2}=\beta e_{1}$, where $\alpha$ and $\beta$ are integers $\neq 0$. He finds systems of integral elements, as defined by Dickson*, when $\alpha$ and $\beta$ are odd and also in one case when $\alpha$ is even and $\beta$ odd.

\footnotetext{
* See his Algebras and their Arithmetics, § 87.
} 
19. Professor F. R. Sharpe: The irrationality of the general cubic variety in four-dimensional space.

It has long been considered probable but it has not been definitely proved that the general cubic variety in fourdimensional space is irrational. In the present paper rational cubic varieties are studied by means of the space involution derivable from the hyperplane sections through a point on the variety, and the conclusion is drawn that in all cases the variety is not the general cubic variety. If this is the case, then the general cubic variety must be irrational.

20. Professor Edward Kasner: Analytic curves for which the chord equals the arc.

The problem is to find regular analytic curves through a given point $O$ such that the arc $O P$ equals the chord $O P$ for every point $P$. In complex space there is a large class of such curves besides the straight lines, which are the only real solutions. In an ordinary three-space the curves lie in minimal planes. Generalization to Riemann space of $n$ dimensions is discussed.

21. Professor J. L. Synge: A generalization of the Riemannian line-element.

This paper outlines the geometry that results from the assumption that the square of the line-element is a homogeneous function of the second degree of the differentials of the coordinates, without the additional hypothesis of Riemann that this function is quadratic. It is found that the equations of the geodesics may be presented in their usual form,

$$
\frac{d^{2} x^{i}}{d s^{2}}+\left\{\begin{array}{c}
i k \\
i
\end{array}\right\} \frac{d x^{j}}{d s} \frac{d x^{k}}{d s}=0,
$$

where the Christoffel symbols contain the coordinates and are homogeneous functions of zero degree in the first derivatives of the coordinates with respect to $s$. The natural generalization of the Levi-Civita formula for parallel propagation of a vector is found to contain the second derivatives of the coordinates with respect to the parameter as well as their first derivatives. The "angle between two vectors with respect to a given curve" and other geometric concepts are discussed. 
22. Professor G. A. Bliss: Boundary value problems for systems of linear differential equations of the first order.

In this paper the author studies the problem of determining the solutions of a system of differential equations $\frac{d u_{i}}{d x}=\sum_{k=1}^{n}\left[A_{i k}(x)+\lambda B_{i k}(x)\right] u_{k}(x)+g_{i}(x), \quad(i=1, \cdots, n)$, with boundary conditions of the form

$s_{i}(u)=\sum_{k=1}^{n}\left[M_{i k} u_{k}(a)+N_{i k} u_{k}(b)\right]=h_{i}, \quad(i=1, \cdots, n)$,

where the coefficients $M_{i k}, N_{i k}$ and $h_{i}$ are constants. A very large number of boundary value problems for ordinary differential equations, hitherto separately studied in the literature, are expressible as special cases of this. It is shown that when the problem is definitely self-adjoint according to a suitable definition the characteristic numbers $\lambda$, are denumerably infinite in number and real, and each has associated with it a unique set of characteristic functions $u_{i \nu}(x)(i=1, \cdots, n)$. Every set of functions $f_{i}(x)(i=1, \cdots, n)$ with suitable continuity properties which satisfy the boundary conditions $s_{i}(f)=0(i=1, \cdots, n)$ is expressible uniquely in the form $f_{i}(x)=\sum_{\nu=1}^{\infty} c_{\nu} u_{i v}(x)(i=1, \cdots, n)$, and the series converges uniformly on the interval $a \leqq x \leqq b$.

23. Professor W. A. Hurwitz: On de la Vallée Poussin summability.

The definition of summability given by de la Vallée Poussin has been studied by Plancherel, C. N. Moore, Gronwall, and Kogbetlianz. In the present paper, the author shows its equivalence to other similar definitions, generalizing results of Kogbetlianz, proves that it permits omission and adjunction of terms, establishes an appropriate necessary condition for summability, and considers the relation of the definition to other standard methods of summability.

24. Professor W. A. Hurwitz: Characteristic parameter. values for an integral equation.

It is well known that the characteristic parameter values for a real symmetric (more generally, complex hermitian) kernel are all real; this result is easily extended to give conditions that the characteristic values lie on any straight 
line through the origin. In this paper, the author gives like criteria for a straight line not through the origin. A typical result is the following: If $\alpha \neq 0$, and if

$$
\begin{aligned}
\bar{\alpha} K(x, y)+\alpha \bar{K}(y, x) & =2 \int K(x, s) \bar{K}(y, s) d s \\
& =2 \int \bar{K}(s, x) K(s, y) d s
\end{aligned}
$$

the characteristic parameter values $\lambda$ for the kernel $K(x, y)$ lie on the straight line $R(\lambda \alpha)=1$.

25. Professor W.A.Hurwitz: A trivial Tauberian theorem.

An example is given of a Tauberian theorem, otherwise unimportant, which exhibits cases of each of the customary Tauberian conditions $\left(\left|u_{n}\right|<K f_{n}, u_{n}<K f_{n}, u_{n}>-K f_{n}\right.$, $\left.u_{n} / f_{n} \rightarrow 0\right)$, and thus throws light on the occurrence of the different types.

26. Professor Marston Morse: Relations "in the large" between the numbers of extremals of different types joining two fixed points.

In this paper the author is concerned with the "regular" plane problem in the non-parametric form. He shows that under certain boundary conditions the problem of the determination of the extremals joining two fixed points is equivalent to the problem of determining the critical points of a function, $f$, of a large but fixed number of variables. On any extremal joining the two given points the number of points conjugate to either end point determines the nature of the corresponding critical point of $f$. In a paper presented to the Society in December, 1923, the author gave a set of relations between the numbers of critical points of different types of a function $f$ under certain boundary conditions. These relations lead at once to a set of relations between the numbers of extremals of the different types joining the two fixed points.

27. Dr. C. R. Adams: An existence theorem for a linear partial difference equation of the intermediate type.

The equation considered is $g(x+1, r y)=a(x, y) g(x, y)$, in which $r$ is a constant, real or complex, not zero and 
in absolute value different from unity; $a(x, y)$ is a given polynomial in $x$ and $y$ (no added generality is secured by taking it a rational function); and $g(x, y)$ is the function to be determined. There exist in general two formal series solutions, the first in powers of $1 / x$ and $y$ and the second in powers of $1 / x$ and $1 / y$. These series do not in general converge. There exist, however, two solutions which are analytic save for poles over the entire $x$ - and $y$-planes ( $y=0$ excluded for the second solution) and which, in a certain sense, are asymptotically represented by the corresponding formal series. Roughly speaking, the sense is this: the first solution is represented by the first formal series with respect to $x$ in any half-plane and with respect to $y$ in the complete neighborhood of the origin; the second is represented asymptotically by the second formal series with respect to $x$ in any right half-plane and with respect to $y$ in the complete neighborhood of infinity.

28. Dr. C. R. Adams: Note on the existence of analytic solutions of non-homogeneous linear q-difference equations, ordinary and partial.

This paper deals with a system $g_{i}(q x)=\sum_{j=1}^{n} a_{i j}(x) g_{j}(x)$ $(i=1,2, \cdots, n-1), g_{n}(q x)=\sum_{j=1}^{n} a_{n j}(x) g_{j}(x)+b(x)$, no loss in generality being suffered in taking only one equation of the system to be non-homogeneous. If the coefficient functions $a_{i j}(x)$ and $b(x)$ are rational and if $|q|$ is $\neq 1$, the system in general possesses two formal series solutions. These series converge in a large class of cases and so represent, in certain regions, analytic solutions. The region of analyticity of the solutions can be extended by repeated use of the system itself. The relation between the two solutions is found to be expressible in terms of solutions of the associated homogeneous system (obtained by deleting $b(x))$ and certain periodic functions the nature of which can, in important instances, be completely determined. Similar theorems are proved for a system analogous to the above but in which the functions involved depend on more than one variable*.

* More precisely, a non-homogeneous system of the class treated by the author in a recent paper The general theory of a class of linear partial q-difference equations, Transactions of THIs SocIETr, vol. 26 (1924), pp. 283-312. 
29. Professor E. R. Hedrick: A generalization of Morera's theorem.

Generalizations of Morera's theorem have been stated by several authors. According to J. Wolff, who gave one such generalization in a paper entitled On sufficient conditions for analyticity at the Toronto Congress, Looman has given generalizations in papers in NIEUW ARchIEF VOOR WISKUNDE (vol. 14, pp. 234-239). The present paper gives still further generalizations. Let us first assume that the real and the imaginary parts of a function of a complex variable have continuous first derivatives, and let us employ the expression analytic at a point in the sense defined by the author and others (Liouville, (9), vol. 2 (1923), pp. 327-342). Then it is shown in the present paper that a necessary and sufficient condition that a function be analytic at a point is that the limit of the ratio of $f(z) d z$ over a square about that point to the area of the square approach zero with the area, on a given sequence of squares. It follows that a necessary and sufficient condition that the function be analytic at every point is that the same property hold at each point of a set everywhere dense in the region.

30. Professor J. F. Ritt: A theorem on rational functions analogous to a theorem of Liiroth.

If $F(z)=\varphi_{1}\left[\varphi_{2}(z)\right]$, all three functions being rational, $\varphi_{2}(z)$ will be called a forefactor, and $\varphi_{1}(z)$ a postfactor of $F(z)$. It is a well known theorem of Lüroth that given any two rational functions, $F_{1}(z)$ and $F_{2}(z)$, there exists a rational combination $\varphi(z)=R\left(F_{1}, F_{2}\right)$ of the two functions, such that $F_{1}(z)$ and $F_{2}(z)$ are both rational functions of $\varphi(z)$. This theorem implies immediately, and is almost equivalent to, the following result: Any two rational functions, $F_{1}(z)$ and $F_{2}(z)$, have a forefactor $\varphi(z)$ in common such that every common forefactor of $F_{1}(z)$ and $F_{2}(z)$ is a forefactor of $\varphi(z)$. The present paper investigates the common postfactors of a pair of functions. A result analogous to the above is obtained, namely that any two rational functions have a common postfactor of which every common postfactor is a postfactor. The writer's proof of this theorem does not resemble that of the theorem of Lüroth. The dissimilarity seems actually to be due to differences in the nature of the theorems. 
31. Professor Olive C. Hazlett: Formal modular covariants as algebraic invariants.

In this paper it is shown how, as a result of a symbolic theory developed several years ago, it follows that any isobaric formal modular invariant of a binary form $f$ with respect to the Galois field, $G F\left[p^{n}\right]$, of order $p^{n}$ is congruent, $\bmod p$, to an algebraic invariant $A$ of the form $f$, where $A$ is a rational integral invariant if $p \neq 2$ and is either a rational integral invariant or a square of such if $p=2$. Another type of formal modular invariant of $f(a ; x, y)$ is an algebraic invariant of $f\left(a^{p^{n}} ; x, y\right)$ and $f(a ; x, y)$. Similarly, the formal modular invariants of $f(a ; x, y)$ are in general algebraic invariants of $f(a ; x, y)$ and certain related forms. By applying these results, the author attempts to determine a general method of finding the formal modular invariants and covariants of a general form from algebraic invariants, which shall be of general applicability.

32. Professor C. de la Vallée Poussin: On functions differentiable an infinite number of times.

This paper discusses (1) general relations between the law of limitation of successive derivatives and that of the Fourier coefficients of a periodic function, and (2) the existence of functions whose successive derivatives take given values at a point.

\section{Professor R. L. Moore: Covering theorems.}

Using the following definition: A point set $K$ is said to be uniformly relatively well imbedded in a set of regions $G$ if there exists a positive number $d$, less than unity, such that each point of $K$ belongs to some region $R$ of $G$ and is at a distance from the boundary of $R$ less than $d$ times the diameter of $R$, the author proves these theorems: (1) If, for each circle $g$ of a set $G, h_{g}$ is a definite circle concentric with $g$ and there exists a positive $e$, less than unity, such that, for every $g$, the radius of $h_{g}$ is less than $e$ times that of $g$, then the set $G$ contains a subset such that, for each $g, h_{g}$ is within one and only one circle of this subset. (2) If $G$ is a set of circular regions and $K$ is a point set of measure zero, and, for every positive $e, K$ is uniformly relatively well imbedded in a subset of $G$ whose regions are of radius less than $e$, then, for every positive $\varepsilon, G$ contains a subset $G^{*}$ which covers $K$, the sum of the areas 
of the regions of $G^{*}$ being less than $\varepsilon$. (3) J. SplawaNeyman's theorem (Fundamenta Mathematicae, vol. 6) remains true if the words "à son intérieur" are omitted.

\section{Professor R. L. Moore: Concerning the separation of} a continuum which does not separate the plane.

A subset $H$ of a point set $K$ is said to separate the point $A$ from the point $B$ in $K$ in the weak sense if $K-H$ contains no continuum which contains both $A$ and $B$. In this paper it is shown that if, in a plane $S, A$ and $B$ are two distinct points of a bounded continuum $K$ no subset of which separates $S$, and $G$ is a collection of subcontinua no one of which separates $A$ from $B$, in $K$, in the weak sense, then, even if there be uncountably many continua in the collection $G$, their sum cannot separate $A$ from $B$, in $K$, in the weak sense.

35. Professor R. L. Moore: Concerning upper semi-continuous collections.

Suppose $G$ is an upper semi-continuous collection of mutually exclusive continua which fill up a plane $S$. In this paper it is shown that, if the continua of the set $G$ are considered as ideal points and the term region is suitably defined, the set of all such ideal points and regions satisfies the set of axioms $\Sigma_{1}$ of the author's paper On the foundations of plane analysis situs, and thus has all the topological properties of an ordinary plane. Thus we have a theory of arcs, simple closed curves, continuous curves, etc., whose elements are continua of the set $G$. For example, a simple closed curve of continua of the set $G$ is a closed and bounded set of such continua which is disconnected by the omission of any two continua of $G$ which belong to it.

36. Mr. H. M. Gehman: A characterization of simple continuous curves.

It is proved that if $M$ is a closed and connected set of points in a euclidean plane, such that $M-W$ is uniformly connected im kleinen relative to $W$, where $W$ is any closed and connected subset of $M$ consisting of more than a single point, then $M$ is a simple continuous arc, a simple closed curve, a ray, or an open curve. 
37. Professor Norbert Wiener: Quasi-analytic functions and quadratic forms.

The author develops an extension of the Denjoy-Carleman theory of quasi-analytic functions, making use of the properties of a certain quadratic form in the Fourier coefficients of a function.

38. Professor Norbert Wiener: The representation of functions by trigonometric integrals. Part 2.

The author develops a method for deducing Bohr's relation between the sum of the squares of the coefficients of a quasi-periodic function and the average of the square of the function from the general theory of the trigonometric expansions of bounded functions.

39. Professor Norbert Wiener: Note on a paper of O. Perron.

The author points out that the methods of solving the Dirichlet problem in its generalized sense, given by 0 . Perron in a recent paper in the Mathematische ZEITsChrift, enable one to build up a generalization of the Dirichlet problem entirely equivalent to that exhibited by the author in two recent papers.

40. Dr. F. H. Murray: On certain families of orbits with arbitrary masses in the problem of three bodies. Second paper.

In this paper a discussion is given of the stability of the straight line and equilateral triangle solutions, respectively, in the problem of three bodies, by means of the results of two preceding papers. It is shown that certain generalized theorems of Bohl can be applied directly to a neighborhood of the straight line solutions, with arbitrary masses, and to a neighborhood of the equilateral triangle solutions, if the masses are such that the characteristic exponents of the generating orbits are not all pure imaginaries; the mutual distances are assumed constant for the generating orbits in both cases.

41. Mr. F. W. Perkins: On the oscillation of harmonic functions.

Given any circle $C$ and a function $u$ finite and harmonic within $C$, let the oscillation of $u$ on $C$ (denoted by $D u \mid c$ ) be defined as the difference between the upper and lower 
bounds of $u$ within $C$. The most important of the new results in the present paper are contained in the following theorems: Let $P_{1}, P_{2}$ be any two points within a circle $C$, and draw the two circles $C_{1}, C_{2}^{r}$ through $P_{1}$ and $P_{2}$ which are tangent to $C$. Denote by $A_{1}, A_{2}$ the angles subtended in these circles by the chord $P_{1} P_{2}$, the vertices being at the points of tangency of $C$ with $C_{1}, C_{2}$ respectively. Let $u$ be any function which is finite and harmonic within $C$. Then $\left|u\left(P_{1}\right)-u\left(P_{2}\right)\right| \leqq\left[\left(A_{1}+A_{2}\right) / \pi\right] D u \mid c$. Let $O$ be the center and $A$ the radius of a circle $C$, and let $P$ be a point interior to $C$, at a distance $\varrho$ from 0 . Let $u$ be any function finite and harmonic within $C$, and denote by $[d u / d \xi]_{P}$ the directional derivative of $u$ at $P$ in an arbitrary direction $\xi$. Then $\left|[d u / d \xi]_{P}\right| \leqq(2 / \pi)\left[A /\left(A^{2}-\varrho^{2}\right)\right] D u \mid c$.

42. Mr. R. M. Foster: The driving-point impedance of an electric circuit.

Necessary and sufficient conditions that a rational function $S(\lambda)$ represent the driving-point impedance of an electric circuit consisting of resistances, inductances, and capacities for oscillations proportional to $e^{\lambda t}$ are determined in this paper for all cases in which the impedance function $S(\lambda)$ is of the first or second degree, by determining (1) the domain of values within which the coefficients of $S$ must lie, (2) the corresponding domain of values for the roots and poles of $S$, and (3) the form of the $S$ curve for real sinusoidal oscillations, that is, for $\lambda=2 \pi f i$, the frequency $f$ increasing from zero to infinity.

43. Professor J. S. Taylor: A note on the theory of depreciation.

In a paper previously presented to the Society, the author attacked the problem of distributing the depreciation charges for a machine having a measurable unit output so that the unit cost of production for an average machine would remain constant (or vary in any preassigned way) during the period of use, and of determinig the period of use so that this unit cost would be a minimum. In the present paper, the problem is that of determining the period of use and distributing the depreciation charges so that the profit shall be a maximum. As before, the method is dependent upon the availability of statistical information concerning the behavior of the type of machine 
in question. Particular attention is called to the dependence of the useful life upon the market value of used machines, with the attendant question of the relative desirability of the purchase of second hand machines instead of new ones.

44. Professor E. W. Chittenden: A simple proof of the topologic invariance of the sets F@ of Hausdorff.

The sets $F_{\varrho} \delta$ of Hausdorff are products of sums of closed sets. S. Masurkiewicz has proved that every set which is homeomorphic with a set $F \varrho \delta$ is also a set $F \varrho \delta$. It is the purpose of the present note to present comparatively simple proofs of this proposition and a related theorem of Sierpinski. This theorem states that the property of being the greatest limit of a sequence of closed sets is also a topologic invariant.

45. Professor A. A Bennett: Generalized elementary symmetric functions.

The plan proposed in a previous paper by the author is here carried out. A study is made of the general linear function in a domain of integrity. By combinatorial methods alone, the usual properties of the elementary symmetric functions are established in this more general case. Thus, in particular, Newton's identities, Waring's formula, and related theorems are proved. By introducing further conditions such as the finite existence of $f(1)$, where $f$ is the linear function, a general norm is defined and the usual properties of the determinant are proved in so far as these are independent of the structure of an assumed matrix. Thus all results are equally valid for the determinant of a linear transformation and the norm of an algebraic number. Incidentally, totally new proofs of Newton's identies and related theorems are given which are elementary although frequently not as compact as the usual discussions. The entire treatment is necessarily independent of any appeal to the conjugates of a given element.

46. Professor A. A. Bennett: Semi-groups.

The notion of semi-group, or of a set with the "associative group property" is fundamental. Recent widespread interest in linear algebras has developed many concepts foreign to the classical group theory but belonging properly to semi-groups. The author examines the semi-group on 
its own account. Important concepts are the idempotent elements and absorbent elements. Thus $a$ absorbs $b$ if $a b=b a=a$. Among the theorems proved are the following: In each semi-group there exists a sequence $a_{1}, a_{2}, \cdots, a_{n}$, where each $a_{i}$ absorbs $a_{i+1}$, and where the sequence cannot be extended by inserting new terms or in other prescribed ways. In such a complete sequence each non-idempotent element is either immediately preceded or immediately succeeded by an idempotent element. If $A$ and $B$ are semigroups, and $A$ is a subset of $B$, such that $A B=B A=A$, $A$ is said to be a normal subset of $B$. It seems possible to accomplish almost or quite as much with this concept of normal subset as with that of invariant subset, while numerous special theorems are shown to hold for normal subsets which are not applicable to the less specific concept of invariant subset.

47. Professor A. A. Bennett: Five axioms for point and translation in affine geometry.

This paper appeared in full in the November-December number of this Bulletin.

48. Professor A. A. Bennett: Biaffine geometry.

In this paper the author introduces biaffine geometry as a subject of study on its own account. Geometrically, it may be described as the affine geometry of vectors, and analytically it is the study of homogeneous non-singular linear transformations, without the use of arbitrary multipliers. This geometry enjoys the same duality manifested by projective geometry, while remaining finite. Thus there is parallelism among points as among lines. Parallel coplanar lines fall into two mutually exclusive separated sets. The vector has a dual, the "sector", analogous to an angle in some respects. Conics fall into many types, some self-dual, others not. Points may be added and subtracted except under certain exceptional restrictions. The present study covers points, lines, and conics only.

49. Professor R. L. Wilder: On connected sets which cut the plane.

Let $M$ be a connected set (containing more than one point) which lies in a plane $S$ and consists entirely of non-cut points but which is disconnected by the omission 
of any two of its points. Under these conditions the author shows that $M$ cuts the plane $S$ in the sense that there exist at least two points, $A$ and $B$, of $S-M$, which do not lie in a subcontinuum of $S-M$. If to the above conditions be added the condition that $M$ is closed, $M$ is, in fact, a simple closed (Jordan) curve.

50. Mr. R. G. Lubben: On the approximate covering of a point set by a finite set of chains.

The author shows that if, in space of one dimension, $G$ is a set of intervals covering a point set $K$, then, for every positive number $e, K$ contains a subset $K_{e}$, of exterior measure less than $e$, such that $K-K_{e}$ is covered by a finite number of non-overlapping finite simple chains whose links are intervals of the set $G$.

51. Professor E. L. Dodd: The frequency law of a function of one variable.

This paper has appeared in full in the January-February issue of this Bulletin.

52. Mr. M. H. Stone: A comparison of the series of Fourier and Birkhoff.

This paper consists of a study of comparative properties of Fourier's series and of the series defined by Birkhoff in volume 9 of the Transactions of THIS Society, with respect to any summable function, and an investigation of the term-by-term derived series of Birkhoff's series.

53. Mr. M. H. Stone: Irregular differential systems of order two and the related expansion problems.

The problem of the irregular expansions arising from differential systems of order two, as described by Birkhoff in volume 9 of the Transactions of this Society, is here discussed by methods which are an extension of those employed in the paper cited.

54. Dr. Philip Franklin: Weierstrass's approximation theorem.

Lebesgue has given a simple proof of the Weierstrass approximation theorem for continuous functions of one variable, but the extensions to more variables involve 
somewhat more complexity. In this note a simple proof is given for the functions of several variables.

55. Mr. Alonzo Church: On irredundant sets of postulates.

A set of postulates is irredundant if the postulates are independent and no one of them can be replaced in the set by a weaker postulate in such a way that none of the implications of the set are lost. A necessary and sufficient condition that a set of postulates be irredundant is that the negatives of every two be contradictory. This paper contains an irredundant set of postulates for the system of positive and negative integers, and this set is shown to be categorical. The general problem is proposed to find irredundant sets of postulates of not too complicated form.

56. Professor Edward Kasner: Null surfaces.

The author means by a null surface one whose $d s^{2}$ vanishes identically. Such surfaces do not exist in three-space, but do exist in space of four or more dimensions. Geometry in a null plane or surface must be distinguished from geometry in a minimal plane or developable. Null $m$-spreads in a given $n$-spread are discussed.

R. G. D. Richardson, Secretary.

\section{A CORRECTION}

BY D. N. LEHMER

On page 401 of volume 8 of this Bulletin (June, 1902), there is a list of errors in Legendre's tables of linear divisors of quadratic forms. The correction stated under the heading III is wrong. The form $172 x+147$ is correct for the form $t^{2}-43 u^{2}$. 